\title{
Magnetic island effects on axisymmetric equilibria
}

\author{
X. Liu, ${ }^{\text {a) }}$ J. D. Callen, ${ }^{\text {b) }}$ and C. C. Hegna ${ }^{\text {c) }}$ \\ Department of Engineering Physics, University of Wisconsin, Madison, Wisconsin 53706-1609
}

(Received 31 December 2003; accepted 2 June 2004; published online 28 September 2004)

\begin{abstract}
The plasma magnetohydrodynamics equilibrium equation in the vicinity of a single, thin magnetic island region is derived. First, the pressure profile in the vicinity of an island is determined using the continuity of transport fluxes across the island assuming a diffusive form for the fluxes. Then, by considering the island-induced magnetic perturbation, the structure of the current density around an island is examined. The modifications of the pressure and current density in the vicinity of a magnetic island lead to an equation that describes the toroidal equilibrium of the plasma. An "effective" axisymmetric Grad-Shafranov equation is constructed by taking a helical average over the toroidal equilibrium. Thus, we can take account of the three-dimensional magnetic island effects for a thin island in a two-dimensional equation. As with the Grad-Shafranov equation, the calculation does not employ a large aspect ratio or small $\beta$ approximation; however, it uses a small island width $(w \ll r)_{s}$ expansion. Finally, we determine the time-varying signal that would be observed on a probe measuring a scalar quantity such as the pressure when a magnetic island structure is rotating relative to the probe.
\end{abstract}

(C) 2004 American Institute of Physics. [DOI: 10.1063/1.1787811]

\section{INTRODUCTION}

In general, the plasma magnetohydrodynamics (MHD) equilibrium in axisymmetric tokamaks is governed by the Grad-Shafranov equation ${ }^{1}$

$$
\Delta^{*} \Psi=-\mu_{0} R^{2} \frac{d P}{d \Psi}-I I^{\prime}
$$

which applies to a two-dimensional (2D) situation. However, nonlinear tearing mode instabilities produce magnetic islands in an axisymmetric toroidal, magnetically confined plasma. Such islands break the axisymmetric property of the toroidal equilibrium and introduce (3D) structure. Rigorously speaking, one should solve a fully 3D set of equations for the magnetic topology. Numerical techniques exist for solving the $3 \mathrm{D}$ equilibrium problem. ${ }^{2}$ However, for a number of reasons, it would be helpful to have a 2D equation with respect to only the 2D axisymmetric coordinates. Then, the plasma MHD equilibrium with a magnetic island can be effectively treated, with a $3 \mathrm{D}$ island structure grafted onto the $2 \mathrm{D}$ equilibrium in the vicinity of the rational surface. The technique addressed in the following will show how a prescribed magnetic island affects the nature of the equilibrium solution in the vicinity of the rational surface.

If a single, isolated magnetic island is thin compared to the radius of its singular surface, its effects can be approximated by a single helically resonant magnetic flux perturbation. A mathematical description of the axisymmetric magnetic equilibrium and perturbations is developed in Sec. II. The effect of the helical perturbation and the magnetic island it induces are localized to the vicinity of the magnetic island, the mathematical properties of which are developed in Sec.

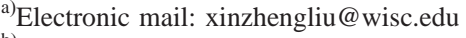

b)Electronic mail: callen@engr.wisc.edu

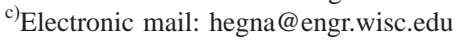

III. In the magnetic island region, the parameters of the plasma such as pressure and current density are generally functions of magnetic flux $\Psi$, helical angle $\alpha$, and poloidal angle $\theta$. After obtaining the profiles and equilibrium equations for these quantities in three dimensions, we average them over the helical angle $\alpha$ at fixed $\Psi$ and $\theta$ to obtain the 2D equation that we desire in Sec. IV. To make the result applicable to various cross-section geometric shapes, the calculations are performed in magnetic flux coordinates. The island-induced modification of the pressure gradient is localized mainly to the magnetic island region. It is worked out in detail in Sec. V. Then, Sec. VI specifies the 3D variations of the pressure (or any scalar quantity) in the vicinity of a magnetic island in terms of what a fixed probe would observe when the plasma rotates toroidally. Finally, the results are summarized in Sec. VII.

\section{AXISYMMETRIC EQUILIBRIUM AND PERTURBATIONS}

To lowest order, the plasma configuration is taken to be an axisymmetric equilibrium. The magnetic field can thus be written as

$$
\mathbf{B}_{0}=I \nabla \zeta+\nabla \zeta \times \nabla \Psi,
$$

where $\mathrm{I}=\mathrm{RB}_{\text {toroidal }}$ is the poloidal current function, $\Psi$ labels the magnetic surfaces of the axisymmetric equilibrium, and $\zeta$ is the (axisymmetric) toroidal angle. A straight-field line poloidal angle $\theta$ is introduced so that

$$
\mathbf{B}_{0}=\boldsymbol{\nabla} \Psi \times \nabla(q \theta-\zeta),
$$

where $q=q(\Psi)$ is the safety factor (toroidal winding number of magnetic field lines). In magnetic flux coordinates, the Jacobian is given by $\sqrt{g}=1 / \nabla \Psi \times \nabla \theta \cdot \nabla \zeta$. In the following, the equilibrium axisymmetric field is taken to be consistent with the Grad-Shafranov equation. 
We consider a magnetic perturbation that is resonant at the rational surface where $q_{0}=m / n$. The helical angle coordinate $\alpha$ is defined as $\alpha \equiv \zeta-q_{0} \theta$. In the vicinity of the magnetic island, some slowly changing quantities can be approximated by Taylor series expansions: $f=f_{0}+f^{\prime} x$, where $x \equiv \Psi-\Psi_{0}$ and $f^{\prime} \equiv d f / d \Psi$ is the derivative with respect to the magnetic flux coordinate.

Without loss of generality, we can introduce magnetic perturbations using a vector potential of the form

$$
\delta \mathbf{A}=A \boldsymbol{\nabla} \theta-\chi \boldsymbol{\nabla} \alpha,
$$

where both $A$ and $\chi$ are of order $x^{2}$ [consistent with the small island approximation, see Eq. (9) below], and the component of $\delta A$ in the $\nabla \Psi$ direction can be eliminated by an appropriate gauge choice. The perturbed magnetic field is thus

$$
\begin{aligned}
\delta B & =\boldsymbol{\nabla} \times \delta A \\
& =\nabla A \times \nabla \theta-\nabla \chi \times \nabla \alpha \\
& =\nabla \zeta \times \nabla \chi+\nabla\left(A+q_{0} \chi\right) \times \nabla \theta .
\end{aligned}
$$

In the vicinity of the rational surface, this magnetic perturbation can produce a magnetic island. Away from this surface, the magnetic surfaces are slightly sinusoidally deformed from their axisymmetric equilibrium values.

\section{PERTURBED MAGNETIC FLUX SURFACE}

The magnetic perturbations introduced via Eq. (5) will change the shape of the magnetic flux surfaces in the vicinity of the magnetic island. The equilibrium magnetic field near the rational surface at $q=q_{0}$ can be expanded as

$$
\mathbf{B}_{0}=\frac{q}{q_{0}} \boldsymbol{\nabla} \Psi \times \nabla\left(q_{0} \theta-\zeta\right)+\nabla \Psi_{0} \times \nabla \zeta,
$$

where $\Psi_{0} \equiv \int d \Psi\left(q / q_{0}-1\right)$. To leading order, $\Psi_{0}=q_{0}^{\prime} x^{2} / 2 q_{0}$.

The perturbed magnetic field can be written as

$$
\delta \mathbf{B}=\boldsymbol{\nabla} \frac{A}{q_{0}} \times \boldsymbol{\nabla} \zeta-\nabla\left(\frac{A}{q_{0}}+\chi\right) \times \nabla \alpha .
$$

For isolated, small magnetic islands, $A$ can be approximated by a single helically resonant harmonic, $A=A_{c} \cos \alpha$. The helical magnetic flux surfaces in the $x-\alpha$ plane, as illustrated in Fig. 1, are defined by $\Psi_{0}+\left(A / q_{0}\right)=$ const, and become

$$
\Psi^{*}=\frac{q_{0}^{\prime} x^{2}}{2}+A_{c} \cos \alpha=C,
$$

in which $\Psi^{*}$ is a helical magnetic flux function, which we will use later.

From Eq. (8), it is easy to show that the total width of the magnetic island in magnetic flux variables is

$$
w=4 \sqrt{A_{c} / q^{\prime}},
$$

in terms of magnetic flux variables, or $w=4 \sqrt{\widetilde{B}_{r} L_{s} / k_{\theta} B_{0}}$ in real space with $k_{\theta}=m / r_{s}$, where $r_{\mathrm{s}}$ is the (cylindrical) radius of the rational surface. The subscript 0 has been dropped for simplicity. In the following we treat the ratio of the magnetic island width to macroscopic length scales as a small number $\left(\delta \equiv w / r_{s} \ll 1\right)$ and use this as an expansion parameter.

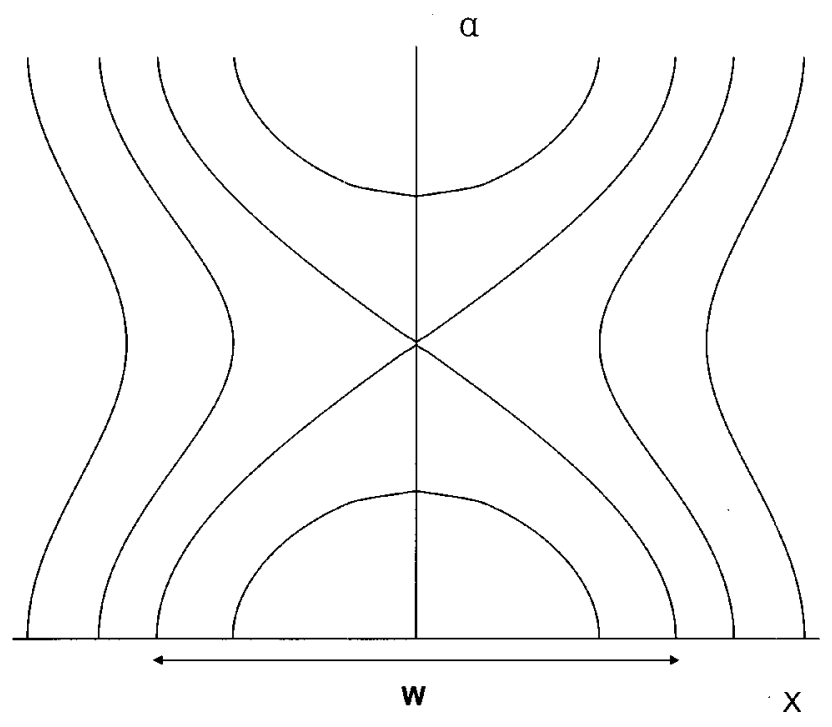

FIG. 1. Contour plot of lines of constant $\Psi^{*}$ (magnetic flux surfaces) from Eq. (8).

Since $\mathbf{B} \cdot \boldsymbol{\nabla} \Psi^{*}=0$ to leading order and MHD force balance requires $\mathbf{B} \cdot \boldsymbol{\nabla} p=0$, we find $p=p\left(\Psi^{*}\right)$.

In what follows, the amplitude of the magnetic island is prescribed and the resulting corrections to the equilibria are computed. A self-consistent calculation of the island width is not sought here but is described in a number of other papers. ${ }^{3}$

\section{MODIFIED GRAD-SHAFRANOV EQUATION}

To obtain the equilibrium relation that generalizes the Grad-Shafranov equation to include the effect of magnetic islands, we calculate the toroidal component of the current density in two ways. First, we obtain $\mathbf{J}$ from Ampère's law by taking the curl of magnetic field (both equilibrium and perturbation parts):

$$
\begin{aligned}
\boldsymbol{\nabla} \zeta \cdot \mathbf{J} & =\frac{1}{\mu_{0}} \boldsymbol{\nabla} \cdot\left[\left(\mathbf{B}_{0}+\delta \mathbf{B}\right) \times \boldsymbol{\nabla} \zeta\right] \\
& =\frac{\Delta^{*}(\Psi+\chi)}{\mu_{0} R^{2}}+\frac{1}{\mu_{0}} \boldsymbol{\nabla} \cdot\left[\frac{\partial\left(A+q_{0} \chi\right)}{\partial \zeta} \frac{\boldsymbol{\nabla} \theta}{R^{2}}\right],
\end{aligned}
$$

where $\Delta^{*}$ is the usual second derivative operator in the GradShafranov equation. ${ }^{1}$

$$
\Delta^{*} \psi \equiv R^{2} \nabla \cdot \frac{1}{R^{2}} \nabla \psi
$$

Second, we decompose $\mathbf{J}$ into two parts: parallel to $\mathbf{B}_{0}$ and perpendicular to it. The lowest order radial force balance is given by

$$
\left(\mathbf{J}_{0}+\delta \mathbf{J}\right) \times \mathbf{B}_{0}=\nabla p .
$$

An additional term with $\delta \mathbf{B}$ is of order $\delta \equiv w / r_{s} \ll 1$ smaller than the terms retained and hence negligible, since $\delta \mathbf{J}=\boldsymbol{\nabla}$ $\times \delta \mathbf{B}$ accounts for the order unity corrections to $\mathbf{J}_{0}$. The total $\mathbf{J}$ is given by its parallel and perpendicular components: 


$$
\mathbf{J}=Q \mathbf{B}_{0}+\frac{\mathbf{B}_{0} \times \boldsymbol{\nabla} p}{B_{0}^{2}}, \quad Q \equiv \frac{\mathbf{J} \cdot \mathbf{B}_{0}}{B_{0}^{2}}=\frac{J_{/ I}}{B_{0}},
$$

where the parallel current in the vicinity of the magnetic island is given by ${ }^{4}$

$$
\mathrm{Q}=\bar{\sigma}-I \frac{\partial p}{\partial \Psi} \frac{1}{B_{0}^{2}}+I \frac{\partial p}{\partial \Psi} \frac{1}{\left\langle\mathrm{~B}_{0}^{2}\right\rangle},
$$

in which $\bar{\sigma}=\left\langle Q B^{2}\right\rangle /\left\langle B^{2}\right\rangle$ is an average of the parallel current function $\mathrm{Q}$ over poloidal angle at fixed $\Psi$ and $\alpha$. Specifically, this operation is defined by

$$
\langle f\rangle(\Psi, \alpha)=\frac{\oint \frac{d \theta}{2 \pi} \sqrt{g} f(\Psi, \alpha, \theta)}{\oint \frac{d \theta}{2 \pi} \sqrt{g}} .
$$

The last two terms in Eq. (13) describe the Pfirsch-Schlüter current present in a toroidal plasma. From this representation of the current we find

$$
\boldsymbol{\nabla} \zeta \cdot \mathbf{J}=\bar{\sigma} \frac{I}{R^{2}}-\frac{\partial p}{\partial \Psi}+\frac{I^{2}}{R^{2}} \frac{\partial p}{\partial \Psi} \frac{1}{\left\langle B_{0}^{2}\right\rangle} .
$$

Equating the two forms of the toroidal component of the current given in Eqs. (10) and (15), we obtain

$$
\begin{gathered}
\frac{\Delta^{*}(\Psi+\chi)}{\mu_{0}}+\frac{R^{2}}{\mu_{0}} \nabla \cdot\left[\frac{\partial\left(A+q_{0} \chi\right)}{\partial \zeta} \frac{\nabla \theta}{R^{2}}\right] \\
=-\frac{\partial p}{\partial \Psi} R^{2}+I^{2} \frac{\partial p}{\partial \Psi} \frac{1}{\left\langle B_{0}^{2}\right\rangle}+\bar{\sigma} I .
\end{gathered}
$$

After averaging over the helical angle $\alpha$ (at constant poloidal angle $\theta$ and axisymmetric flux surface $\Psi$, i.e., at constant $\theta$, $x$ ), this will yield the desired thin-island-modified GradShafranov equation. As with the Grad-Shafranov equation, the pressure $p(\Psi)$ and current $\bar{\sigma}(\Psi)$ functions are at this point arbitrary.

In order to calculate the two profile quantities $\partial p / \partial \Psi$ and $\bar{\sigma}$ that are consistent with a slowly growing magnetic island, we need to consider the appropriate transport equations in the vicinity of the island to accurately describe the self-consistent behavior of the plasma. ${ }^{4}$ For the current, we consider a parallel Ohm's law in the form

$$
-\mathbf{B} \cdot \frac{\partial \mathbf{A}}{\partial t}-\mathbf{B} \cdot \boldsymbol{\nabla} \phi=\eta Q B^{2}-\frac{1}{n e} \mathbf{B} \cdot \boldsymbol{\nabla} \cdot \Pi,
$$

where the first two terms are the parallel electric field $\mathbf{E} \cdot \mathbf{B}, \varphi$ is the electrostatic potential, $\eta$ is the plasma resistivity, and $\Pi$ is the electron viscous stress tensor. Using a neoclassical closure for the viscous term, the flux-surface-averaged Ohm's law corresponding to the axisymmetric equilibrium is

$$
\frac{\langle E \cdot B\rangle}{\left\langle B_{0}^{2}\right\rangle}=\eta \bar{\sigma}_{0}+\frac{\mu_{e}}{\nu_{e}} \eta\left(\bar{\sigma}_{0}+\frac{p_{0}^{\prime} V^{\prime} I}{\left\langle B_{0}^{2}\right\rangle}\right),
$$

where the last term comes from a neoclassical closure of the electron viscosity. Specifically, it describes damping of the poloidal electron flow. The poloidal electron flow can be decomposed into a contribution from the parallel current and a contribution from the diamagnetic current. These currents yield the neoclassical correction to the Spitzer resistivity and bootstrap current, respectively. Here $\mu_{\mathrm{e}}$ is the poloidal electron flow damping rate and $\nu_{\mathrm{e}}$ is the electron collision frequency.

We expand the electrostatic potential $\phi$ in the small expansion parameter $\delta \equiv w / r_{s} \ll 1$. The lowest order equation gives $\phi^{0}=\bar{\phi}^{0}$. To next order, the parallel Ohm's law can be written as

$$
\begin{gathered}
-\left\langle\mathbf{B}_{0} \cdot \frac{\partial \overline{\mathbf{A}}_{0}}{\partial t}\right\rangle-\frac{1}{V^{\prime}} \frac{\partial \bar{A}}{\partial t}-\frac{1}{V^{\prime}}\left[\bar{\Psi}^{*}, \phi\right] \\
=\eta_{n c}\left(\bar{\sigma}\left\langle B_{0}^{2}\right\rangle+\frac{\mu_{e}}{\mu_{e}+\nu_{e}} I \frac{\partial p}{\partial \Psi}\right),
\end{gathered}
$$

where $\eta_{n c}=\eta\left(1+\mu_{e} / \nu_{e}\right)$ is the neoclassical resistivity and

$$
[C, D] \equiv \frac{\partial C}{\partial x} \frac{\partial D}{\partial \alpha}-\frac{\partial C}{\partial \alpha} \frac{\partial D}{\partial x} \text {. }
$$

Applying an average over the helical magnetic surface $\Psi^{*}=$ const[from Eq. (8)],

$$
\langle\bar{f}\rangle_{*}=\frac{\oint \bar{f}\left(\bar{\Psi}^{*}, \alpha\right)\left(1 / \partial \bar{\Psi}^{*} / \partial \Psi\right) d \alpha}{\oint\left(1 / \partial \bar{\Psi}^{*} / \partial \Psi\right) d \alpha},
$$

to Eq. (19), an equation for the flux-surface-averaged parallel current profile is obtained:

$$
\langle\bar{\sigma}\rangle_{*}=\bar{\sigma}_{0}-\frac{1}{\eta_{n c} V^{\prime}\left\langle B_{0}^{2}\right\rangle}\left\langle\frac{\partial \bar{A}}{\partial t}\right\rangle_{*}-\left\langle\frac{\partial \delta p}{\partial \Psi}\right\rangle \frac{\mu_{e}}{\mu_{e}+\nu_{e}} \frac{I}{\left\langle B_{0}^{2}\right\rangle},
$$

where $\delta p=p\left(\Psi^{*}\right)-p_{0}-p_{0}^{\prime} x$.

Calculating the precise form for $\bar{\sigma}_{0}$ requires a detailed description of the plasma force balance in the island region. Details of this calculation are given in Ref. 4. Using Eq. (21) and the result from Ampère's law [Eq. 33 in Ref. 4], we obtain

$$
\bar{\sigma}=\frac{q_{0}^{\prime}}{G p_{0}^{\prime}} \frac{d p}{d \bar{\Psi}^{*}}\left[-x q_{0}^{\prime}(E+F)+H \frac{\partial \bar{A}}{\partial \Psi}\right]+\varphi\left(\bar{\Psi}^{*}\right),
$$

where the parameters $E, F$, and $H$ are standard measures of interchange instability physics ${ }^{5}$ and $G \equiv V^{\prime}\left\langle B^{2} /|\nabla \Psi|^{2}\right\rangle$. The function $\phi$ can be deduced by comparing Eqs. (21) and (22). The parallel current profile can finally be expressed as

$$
\begin{aligned}
\bar{\sigma}= & \frac{q_{0}^{\prime}}{\bar{G} p_{0}^{\prime}} \frac{d p}{d \bar{\Psi}^{*}}\left[(E+F) q_{0}^{\prime}\left(\langle x\rangle_{*}-x\right)\right. \\
& \left.+H\left(\frac{\partial \bar{A}}{\partial \Psi}-\left\langle\frac{\partial \bar{A}}{\partial \Psi}\right\rangle_{*}\right)\right]+\langle\bar{\sigma}\rangle_{*} .
\end{aligned}
$$

The first terms represent Pfirsch-Schlüter currents that vary within the helical flux surfaces. The last term, the fluxsurface-averaged parallel current, is obtained from Ohm's law, Eq. (21). 
The solutions for the equilibrium condition for the critical layer encompassing the island region should be matched asymptotically to the exterior region away from the rational surface. The large $|x|$ limit solution for $\bar{A}$ has the form

$$
\bar{A} \approx A_{l}|x|^{\alpha_{l}}+A_{s}|x|^{\alpha_{s}},
$$

where the Mercier indices are defined by

$$
\alpha_{l, s}=\frac{1}{2} \mp \sqrt{-D_{I}}
$$

with $D_{I}=E+F+H-\frac{1}{4}$. Matching gives the perturbed part of the parallel current function $\bar{\sigma}$ :

$$
\begin{aligned}
\delta \bar{\sigma}= & -\frac{1}{\eta_{n c}\left\langle B^{2}\right\rangle \sqrt{g}}\left\langle\frac{\partial \bar{A}}{\partial t}\right\rangle_{*}-\left\langle\frac{\partial \delta p}{\partial x}\right\rangle_{*} \frac{\mu_{e}}{\mu_{e}+\nu_{e}} \frac{I}{\left\langle B^{2}\right\rangle} \\
& +\frac{q_{0}^{\prime}}{\bar{G} p_{0}^{\prime}}\left[\left\langle\frac{\partial \delta p}{\partial x}\right\rangle_{*}-\frac{\partial \delta p}{\partial x}\right] \frac{D_{R}}{\alpha_{s}-H},
\end{aligned}
$$

with $D_{R} \equiv E+F+H^{2}$. Also, the axisymmetric equilibrium part of $\bar{\sigma}$ is given by $\bar{\sigma}_{0}=-I^{\prime}-\left(I p_{0}^{\prime}\left\langle B_{0}^{2}\right\rangle\right)$.

Substituting these results into Eq. (16) and averaging over the helical angle $\alpha$ at fixed $x$ (radial position in the terms of the axisymmetric magnetic flux function) and $\theta$ [an average indicated by subscript effective (eff)], we can obtain the equation we desire. The second term on the left is in the form of $\partial f / \partial \zeta$, whose integral over a $2 \pi$ period of $\alpha$ will be zero. The result of this helical average at constant $x$ is

$$
\begin{aligned}
\frac{\Delta^{*}\left(\Psi+\langle\chi\rangle_{\alpha}\right)}{\mu_{0}}= & -\frac{\partial p_{e f f}}{\partial \Psi} R^{2}-I I^{\prime} \\
& +\left(\frac{q_{0}^{\prime} I}{\bar{G} p_{0}^{\prime}} \frac{D_{R}}{\alpha_{s}-H}-\frac{\mu_{e}}{\mu_{e}+\nu_{e}} \frac{I^{2} \overline{\sqrt{g}}}{\overline{\sqrt{g} B_{0}^{2}}}\right)\left\langle\frac{\partial \delta p}{\partial x}\right\rangle_{* e f f} \\
& +\left(\frac{I^{2} \overline{\sqrt{g}}}{\overline{\sqrt{g} B_{0}^{2}}}-\frac{q_{0}^{\prime} I}{\bar{G} p_{0}^{\prime}} \frac{D_{R}}{\alpha_{s}-H}\right)\left(\frac{\partial p_{e f f}}{\partial \Psi}-p_{0}^{\prime}\right) \\
& -\frac{I}{\eta_{n c} \overline{\sqrt{g} B_{0}^{2}}} \frac{\partial A_{c}}{\partial t} J_{3}
\end{aligned}
$$

where we have defined the following dimensionless integral (see the Appendix):

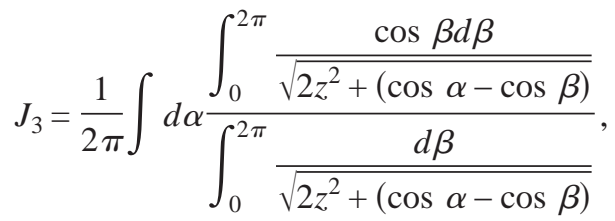

in which $z \equiv x /(w / 2)$ is a dimensionless "radial" magnetic island coordinate $-z \ll 1$ is deep inside island, $z=1$ is on separatrix, and $z>1$ is outside island.

In Eq. (27), $\Psi+\langle\chi\rangle_{\alpha}=\Psi+(1 / 2 \pi) \int_{0}^{2 \pi} \chi d \alpha \equiv \Psi_{\text {eff }}$ labels the island-modified magnetic flux surfaces in the $\Psi-\theta$ cross section. We can see the term on the left and the first two terms on the right correspond to the usual Grad-Shafranov equation in Eq. (1), while the last three terms can be taken as some corrections to the usual equation. In the following sec- tion, the pressure profile is calculated. Then, $\partial p_{e f f} / \partial \Psi$ and $\langle\partial \delta p / \partial x\rangle_{* e f f}$ will be related to $p_{0}^{\prime}$, the radial pressure gradient in the absence of a magnetic island. Thus, all the terms on the right of Eq. (27) are specified in terms of the original (before island) axisymmetric magnetic flux surfaces $\Psi$.

\section{PRESSURE PROFILE}

In the magnetic island region, the pressure is constant on a perturbed magnetic flux surface $\Psi^{*}=$ const. Thus, its profile is a function of the helical magnetic flux label $\Psi^{*}$ in Eq. (8): $p=p\left(\Psi^{*}\right)$. Within the separatrix, we take the pressure to be a constant, at its value on the separatrix. The helical magnetic flux $\Psi^{*}$ is of order $x^{2}$, and $\partial^{*} / \partial \alpha$ is also of order $x^{2}$; however, $\partial \Psi^{*} / \partial x$ is of order $x$. Since we are assuming the magnetic island to be thin, we can assume there are no sources or sinks of particles in the vicinity of the magnetic island and make an approximation that the transport fluxes across it are continuous; hence, $\int \boldsymbol{\nabla} p \cdot \mathbf{d} \mathbf{S}=$ const., in which the integral is performed at constant $\Psi^{*}$ (see Ref. 6). To the lowest order, this quantity becomes

$$
\begin{aligned}
\int \boldsymbol{\nabla} p \cdot \mathbf{d} \mathbf{S} & =\frac{d p}{d \Psi^{*}} \sqrt{g} \nabla^{2} x \int \frac{d \Psi^{*}}{d x} d \theta d \alpha \\
& =\frac{d p}{d \Psi^{*}} \sqrt{g} \nabla^{2} x \int \pm \sqrt{2 q^{\prime}\left(\Psi^{*}-A_{c} \cos \alpha\right)} d \theta d \alpha,
\end{aligned}
$$

and we have

$$
\frac{\partial p}{\partial x}=\left.\frac{d p}{d \Psi^{*}}\right|_{\Psi^{*}=q^{\prime} x^{2} / 2+A_{c} \cos \alpha} \frac{\partial \Psi^{*}}{\partial x} .
$$

Matching with the pressure gradient $p_{0}^{\prime}$ far away from the island, we obtain

$$
\begin{aligned}
& \frac{d p}{d \Psi^{*}}=\frac{2 \pi p_{0}^{\prime}}{\int_{0}^{2 \pi} \pm \sqrt{2 q^{\prime}\left(\Psi^{*}-A_{c} \cos \beta\right)} d \beta}, \\
& \frac{\partial p}{\partial x}=\frac{2 \pi|z| p_{0}^{\prime}}{\int_{0}^{2 \pi} \sqrt{z^{2}+\frac{1}{2}(\cos \alpha-\cos \beta)} d \beta} .
\end{aligned}
$$

Averaging over $\alpha$ at fixed $x$, we obtain the effective value of the pressure gradient in the vicinity of the magnetic island:

$$
\frac{d p_{e f f}}{d x}=p_{0}^{\prime} \int \frac{|z| d \alpha}{\int_{0}^{2 \pi} \sqrt{z^{2}+\frac{1}{2}(\cos \alpha-\cos \beta) d \beta}} \equiv p_{0}^{\prime} J_{1},
$$

in which $J_{1} \equiv J_{1}(z)$ is a dimensionless integral (see the Appendix). The dimensionless integral $J_{1}$ is plotted in Fig. 2. Also, using Eq. (32) we can obtain $\langle\partial \delta p / \partial x\rangle_{* e f f}=p_{0}^{\prime} J_{2}$, in which (see the Appendix) 


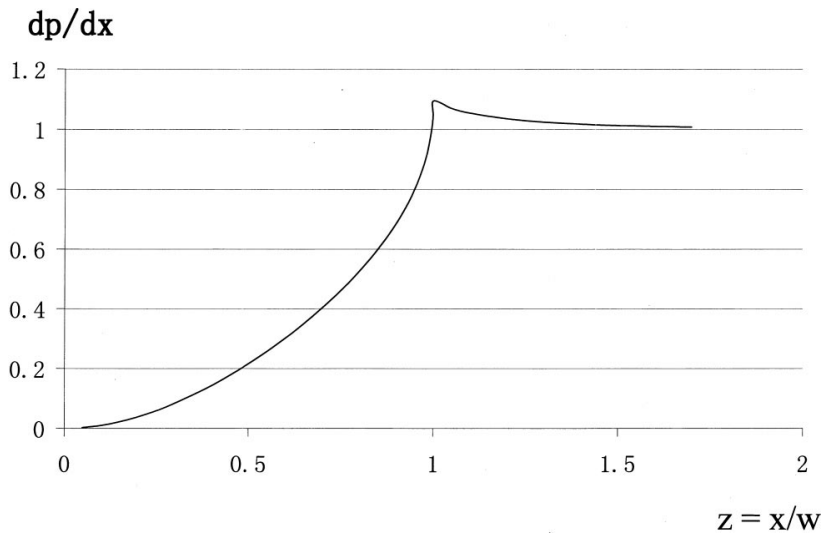

FIG. 2. Effective pressure profile function in the presence of a magnetic island.

$$
\begin{gathered}
J_{2} \equiv-1+2 \pi \int d \alpha /\left[\int_{0}^{2 \pi} \sqrt{2 z^{2}+(\cos \alpha-\cos \beta)} d \beta\right. \\
\left.\cdot \int_{0}^{2 \pi} \frac{d \beta}{\sqrt{2 z^{2}+(\cos \alpha-\cos \beta)}}\right] .
\end{gathered}
$$

The final, island-modified Grad-Shafranov equation, Eq. (27), can be written in terms of the dimensionless integrals $J_{1}, J_{2}$, and $J_{3}$ as

$$
\begin{aligned}
\frac{\Delta^{*} \Psi_{e f f}}{\mu_{0}}= & -\frac{\partial p_{e f f}}{\partial \Psi} R^{2}-I I^{\prime} \\
& +\left(\frac{q_{0}^{\prime} I}{\bar{G} p_{0}^{\prime}} \frac{D_{R}}{\alpha_{s}-H}-\frac{\mu_{e}}{\mu_{e}+\nu_{e}} \frac{I^{2} \sqrt{g}}{\sqrt{g} B_{0}^{2}}\right) p_{0}^{\prime} J_{2} \\
& +\left(\frac{I^{2} \sqrt{g}}{\overline{\sqrt{g} B_{0}^{2}}}-\frac{q_{0}^{\prime} I}{\bar{G} p_{0}^{\prime}} \frac{D_{R}}{\alpha_{s}-H}\right) p_{0}^{\prime}\left(J_{1}-1\right) \\
& -\frac{I}{\eta_{n c} \sqrt{g} B_{0}^{2}} \frac{\partial A_{c}}{\partial t} J_{3} .
\end{aligned}
$$

The small $[z=x /(w / 2) \ll 1$, deep inside the island] and large $[z=x /(w / 2) \gg 1$, far outside the island $]$ limits of the dimensionless integrals $J_{1}, J_{2}$, and $J_{3}$ are discussed in the Appendix.

Equation (35) is the final magnetic-island-modified Grad-Shafranov equation we have been seeking. Comparing with Eq. (1), the right of Eq. (35) is already known (in terms of the original axisymmetric flux surfaces), after we obtain the equilibrium quantities by solving the usual GradShafranov equation and introduce a magnetic island structure and its effects. We only need to solve for $\Psi_{\text {eff }}$ to obtain the new island-modified magnetic flux surfaces.

Since far away $(z \gg 1)$ from the magnetic island all the corrections to the regular axisymmetric Grad-Shafranov equation [Eq. (1)] scale as $1 / z^{4}$, the island-induced corrections to the Grad-Shafranov equation here scale as $\left(w / r_{s}\right)^{4}$; hence they are quite small far from the island. In the vicinity of the magnetic island $(|z|<1)$ the corrections are of order unity - see the following section for the order unity modification of the local pressure gradient. However, because the net change in the pressure gradient is localized to the island region and the Shafranov shift $\Delta(r)$ is determined from an integral over the local pressure profile, one can anticipate ${ }^{7}$ that the island-induced change in the radial position of the rational surface will be of order $\left(w / r_{s}\right)^{2} \ll 1$.

\section{PRESSURE DIAGNOSTIC}

In a tokamak, the plasma usually rotates around the toroidal axis of symmetry. A local plasma parameter probe ${ }^{8}$ at a fixed position will see the pressure at fixed $x$ and changing $\alpha$. To be specific, $\alpha \equiv \alpha_{0}+\omega t$ will range from 0 to $2 \pi$. We can work out the pressure function that the probe sees as follows. Integrating Eq. (31) over an appropriate range of helical magnetic flux surfaces $\left(\Psi^{*}=\right.$ const), we obtain

$$
p(x, \alpha)=p_{s}+\int_{A_{c}}^{\Psi^{*}} \frac{2 \pi p_{0}^{\prime}}{\int_{0}^{2 \pi} \pm \sqrt{2 q^{\prime}\left(\Psi^{* \prime}-A_{c} \cos \beta\right)} d \beta} d \Psi^{*,},
$$

where $p_{s}$ is the pressure within the separatrix. Substituting Eq. (8) into Eq. (36), we can obtain the pressure at a point $(x, \alpha)$. When $\alpha$ goes from 0 to $2 \pi$, the range of pressure variation is

$$
p_{\min }=p_{s}+\int_{A_{c}}^{\left(q^{\prime} x^{2} / 2\right)-A_{c}} \frac{ \pm 2 \pi p_{0}^{\prime}}{\int_{0}^{2 \pi} \sqrt{2 q^{\prime}\left(\Psi^{* \prime}-A_{c} \cos \beta\right)} d \beta} d \Psi^{* \prime}
$$

to

$$
\begin{aligned}
p_{\max }= & p_{s} \\
& +\int_{A_{c}}^{\left(q^{\prime} x^{2} / 2\right)+A_{c}} \frac{ \pm 2 \pi p_{0}^{\prime}}{\int_{0}^{2 \pi} \sqrt{2 q^{\prime}\left(\Psi^{* \prime}-A_{c} \cos \beta\right)} d \beta} d \Psi^{* \prime} .
\end{aligned}
$$

When $x<w / 2$, the lower limit is $p_{s}$.

\section{DISCUSSION AND SUMMARY}

In this paper we have developed procedures for including the effect of a "thin" $\left(w \ll r_{s}\right)$ magnetic island in an otherwise axisymmetric equilibrium. The calculation concentrates on the modification to the equilibrium that occurs in the vicinity of the rational surface. The final island-modified Grad-Shafranov equation is given by Eq. (35). The islandmodified helically averaged magnetic flux surfaces are the $\Psi_{\text {eff }}$ surfaces, which become the axisymmetric flux surfaces $\Psi$ in the absence of a magnetic island. After the $\Psi_{\text {eff }}$ surfaces in the presence of a magnetic island are determined, local-probe-measured (3D) variations in the pressure (or any other scalar plasma parameter that is constant along magnetic field lines) in a toroidally rotating plasma can be determined from Eq. (36).

The structure of the modified Grad-Shafranov equation has been obtained following an averaging procedure. Namely, the island-modified "flux surface" shape is deter- 
mined by two modified profile functions $\partial p_{e f f} / \partial \psi$ and $I I_{e f f}^{\prime}$. The construction of these two profiles for a slowly evolving magnetic island required the specification of the transport equations in the vicinity of the island. For the pressure profile, a constant thermal flux assumption was used with a diffusive Fick's law form for the thermal flux. For the modified current profile, a neoclassical Ohm's law was used that accounts for resistive diffusion as well as neoclassical viscosity. While the actual magnetic topology is described by helical flux surfaces, approximate "axisymmetric" equilibrium profiles have been obtained by averaging the relevant equations over the helical angle. The island-induced changes in the Grad-Shafranov equation are of order unity in the vicinity of the island, but of order $\left(w / r_{s}\right)^{4} \ll 1$ far from the island. The resultant changes in the radial positions of the axisymmetric flux surfaces can be anticipated to be of order $\left(w / r_{s}\right)^{2}$ near the rational surface and $\left(w / r_{s}\right)^{4}$ far from it. However, detailed numerical solutions of the island-modified GradShafranov equation should be performed to confirm these scalings and determine the relevant magnitudes of these island-induced effects on the magnetic flux surface positions.

\section{ACKNOWLEDGMENTS}

This work was supported by the U.S. Department of Energy Grant No. DE-FG02-92ER54139.

\section{APPENDIX: PROPERTY OF INTERGRALS IN THIS WORK}

In Eqs. (33), (34), and (28), we defined three dimensionless integrals $J_{1}, J_{2}$, and $J_{3}$ that are only functions of the variable $z \equiv x /(w / 2)$, which is a dimensionless measure of the radial distance from the rational surface in terms of the half-width of the magnetic island. We will show some of their asymptotic scaling properties here. In all of them the integrals over $\beta$ are performed over an entire period of $2 \pi$, while the integrals over $\alpha$ are only done over the region where the point $(z, \alpha)$ is outside the separatrix. This guaran- tees that $2 z^{2}+(\cos \alpha-\cos \beta)>0$, which yields the limit

$$
|\alpha| \leqslant \cos ^{-1}\left(1-2 z^{2}\right)
$$

for $z$ less than 1 ; if $z$ is greater than 1 , the limit for $\alpha$ is the whole period of $2 \pi$.

First, we examine the limit $|z| \gg 1$-far from the magnetic island. Since the last three terms in Eq. (35) represent the modification to the Grad-Shafranov equation, they must vanish when $z$ is much greater than unity. Specific examinations show that for large $z$

$$
J_{1} \approx 1+\frac{1}{16 z^{4}}, \quad J_{2} \approx-\frac{1}{32 z^{4}}, \quad J_{3} \approx \frac{1}{8 z^{2}},
$$

which satisfy the requirement that Eq. (35) reduces to usual Grad-Shafranov equation, Eq. (1), in the infinite $z$ (far from the magnetic island) case.

We can also obtain an analytical approximation for the three integrals in the small $z$ limit. The limit for $\alpha$ will then be $|\alpha| \leqslant 2 z$. The integral over $\beta$ can be expressed in the form of elliptic integrals of the first and second kind. Using approximation formulas for them, we can obtain for small $z$,

$$
J_{1} \approx z^{2}, \quad J_{2} \approx-\frac{\pi z}{2 \ln z}-1, \quad J_{3} \approx \frac{2 z}{\pi}\left(1+\frac{2}{\ln z}\right) .
$$

${ }^{1}$ J. P. Freidberg, Ideal Magnetohydrodynamics (Plenum, New York, 1987); J. D. Callen and R. A. Dory, Phys. Fluids 15, 1523 (1972).

${ }^{2}$ A. Reiman and H. Greenside, Comput. Phys. Commun. 43, 157 (1986).

${ }^{3}$ P. H. Rutherford, Phys. Fluids 16, 1903 (1973); R. B. White, D. A. Monticello, M. N. Rosenbluth, and B. V. Waddel, Phys. Fluids 20, 800 (1977).

${ }^{4}$ C. C. Hegna, Phys. Plasmas 6, 3980 (1999).

${ }^{5}$ A. H. Glasser, J. M. Greene, and J. L. Johnson, Phys. Fluids 18, 875 (1975).

${ }^{6}$ Z. Chang and J. D. Callen, Nucl. Fusion 30, 219 (1990); C. C. Hegna and J. D. Callen, Phys. Fluids B 4, 4072 (1992).

${ }^{7}$ J. M. Greene and M. S. Chance, Nucl. Fusion 32, 453 (1981); R. L. Miller, M. S. Chu, J. M. Greene, Y. R. Lin-Liu, and R. E. Waltz, Phys. Plasmas 5, 973 (1998); C. C. Hegna, ibid. 7, 3921 (2000).

${ }^{8}$ C. Ren, J. D. Callen, T. A. Gianakon, C. C. Hegna, Z. Chang, E. D. Fredrickson, K. M. McGuire, G. Taylor, and M. C. Zarnstorff, Phys. Plasmas 5, 450 (1998). 